\title{
Health Diagnosis Method of Power Distribution Equipment Based on Holographic Time-scalar Measurement Data
}

\author{
Bing Chen ${ }^{1, \mathrm{a}}$ Ren Wang ${ }^{1, \mathrm{~b}} \quad$ Yang Zhou $^{2, \mathrm{c},{ }^{*}} \quad$ Chenhui Peng ${ }^{2}$ \\ ${ }^{1}$ State Grid Zhenjiang Power Supply Company, Zhenjiang 212001, Jiangsu, China \\ ${ }^{2}$ China Realtime Database Co.,Ltd., Nanjing 210012, Jiangsu, China \\ a13921598100@139.com, bwr921@sina.com, czhy1990@yeah.net,
}

\begin{abstract}
With the continuous expansion of the distribution network, resulting in a large amount of running data. The integration of Realtime Database and distribution network system make it prossible to record distribution holographic scalar measurement data. This paper studied the deep integration of Realtime Database and distribution network system. On the basis of integration, we studied health diagnosis method of power distribution equipment and we carry out in-depth data mining on the distribution history data and model information. The equipment state diagnosis model and the evaluation system are established by cluster analysis, linear regression algorithm and entropy method, and the equipment fault assessment and early warning analysis are realized. The weak links in distribution network can be found in time to ensure the safe and stable operation of the distribution equipment.
\end{abstract}

Keywords: Holographic time-scalar measurement data, Realtime database, Deep integration, State diagnosis, Linear regression, Entropy method

\section{Introduction}

As a power supply to the client network, distribution network is an important infrastructure to ensure social development[1]. In this paper, we first study the deep integration of realtime database[2] and intelligent distribution network dispatching technology support system (D5200)[3,4,5], and realize the transparent access of time series library in storage and distribution automation system. On the basis of the above, the paper studies the health status detection and diagnosis technology of the distribution network equipment, and combines the safety evaluation standard of the 5A level of the distribution network to study the safety evaluation of the plant, the line, the primary equipment and the terminal, and combines the historical sampling data of the equipment at the same time and the historical fault alarm information to create an algorithmic model and find the weak link in the distribution network, given the purpose of fault analysis and early warning.

\section{Realtime Database}

\subsection{Introduction of Realtime Database}

Whether for the traditional industrial automation or emerging Internet of things, cloud computing applications in all areas, the data have an unparalleled importance. Although the application of relational database is very broad, mainly to store business data, there are some bottlenecks in the processing capacity, storage space and query ability, which leads to the limited real-time processing 
ability. The relational database cannot record high-speed sampling data real change process and cannot analysis accurately because the sampling accuracy is not enough.

The realtime database system has the advantages of fast recording speed, high retrieval efficiency and large compression data rate, which can effectively make up for the lack of relational database, to meet the traditional industrial automation and cloud computing and other fields of real-time data processing applications in real time requirements.

\subsection{Integration of realtime database and D5200}

Based on the experience of time series database and intelligent grid dispatching technology support system (D5000), this paper proposes the depth integration scheme of realtime database and D5200. This scheme includes database environment transformation, unified data access technology and distribution network SCADA transformation. The integration of realtime database and D5200 system is completed, which realizes the accurate and complete record and preservation of the data of distribution network operation, and provides functional modules such as real-time sampling definition, sampling query and fine historical data curve display.

D5200 data sources include real-time data collection, manual number and calculation of data. The D5200 system write the above data into the memory database and realtime database at the same time, as Fig.1 shown.

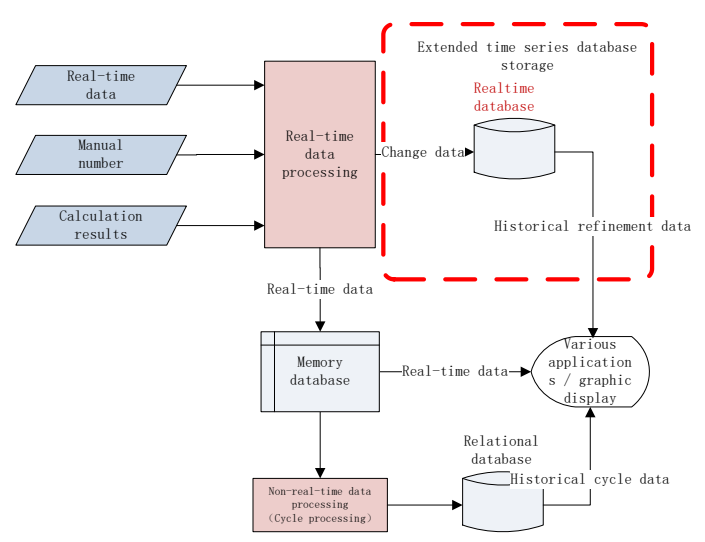

Fig.1 Data storage diagram

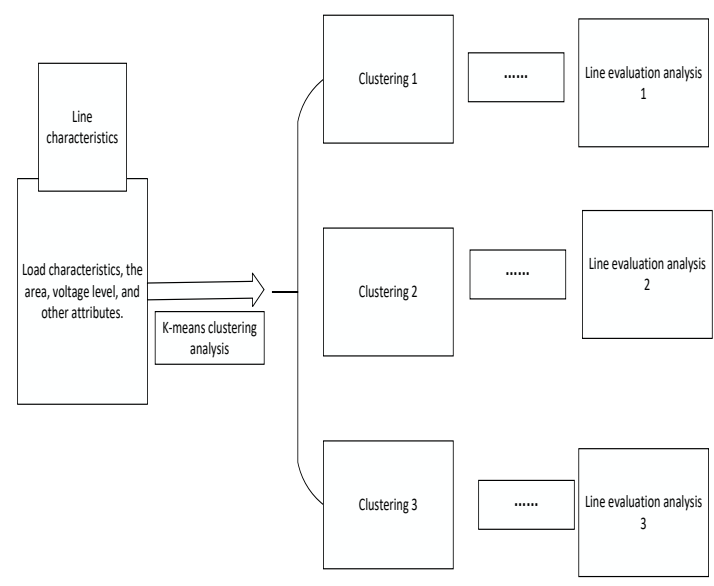

Fig.2 Line classification analysis

We encapsulate the realtime database storage, query and management in the existing D5200 platform layer to provide a unified access interface, including the status monitoring, sampling point definition, data submission and data query. The visualization of the system, such as graphics, curve tools, etc., can directly use the data in the realtime database, and other applications can easily integrate the time series database data source through an interface or service that is added at the platform layer.

\subsection{Realtime database provides data support}

Realtime database mainly stores real-time historical data of all telemetry telegrams and calculated values, abandoning the periodic sampling mode in the traditional D5200 system, recording holographic time-scalar measurement data when it changed which can record the grid operating trajectory more accurately and completely. Realtime database provides a strong and high-precision data support for the next equipment health status diagnosis analysis.

\section{Diagnosis and analysis of health status of distribution network equipment}

\subsection{Equipment status}

The equipment state is a comprehensive measure of the current performance of the various reactions, divided into four levels (normal, attention, abnormal, serious) [4,5]. The status of 
equipment directly or indirectly reflects the health of the equipment and is the prerequisite of the diagnosis of the equipment health status. Therefore, the primary task of equipment health status diagnosis is to collect and process the equipment status. In this paper, we carry out in-depth data mining on the distribution equipment status mainly from the aspect of running information, and finally get the analysis results[6,7]. Based on the complete data of the realtime database, this paper focuses on the evaluation and analysis of the distribution line equipment. The following information is mainly considered in the running information: line load rate, terminal on-line rate, the correct rate of remote action, line connection rate.

\subsection{Diagnosis of equipment health status diagnosis}

\subsubsection{Equipment status evaluation process}

In this paper, K-means clustering[9] of distribution equipment is analyzed according to the different characteristic attributes of equipment, then the operation status of the equipment is evaluated according to the corresponding model algorithm as Fig.2 shown.

Taking the line as an example, K-means clustering analysis is carried out according to the attribute characteristics such as load characteristic, region or voltage level. After the classification is completed, the same evaluation dimension is adopted for different types of lines.

After the classification of the line, a reasonable fault level prediction model is established for each type of line equipment through the linear regression model. The feature parameter information is obtained from the realtime database, and the fault level prediction model is established by SVM linear regression model algorithm. Then we obtain the current information of the current distribution equipment data, after substituting the regression model, the level of equipment failures that may occur to predict. According to the evaluation results, priority is given to overhauling equipment with higher fault level.

For each state indicator, the interval model is established, divided into four levels, normal, attention, abnormal and serious. Each state corresponds to a different range of indicators, and also has a different weight $\mathrm{k}$, the score of each state indicator is $M_{i}=100 \times k$ (1). A device has $\mathrm{n}$ status indicators, depending on the different dimensions, set different weights $C_{i}(i=1,2, \ldots n$ $\left.\sum_{i=1}^{n} C_{i}=1\right)$. The weight value is calculated by the entropy method [10].

The final score of the device is divided into two ways:

1) If there is a certain class or a class of indicators of the state is abnormal or serious, the final score of the device is the minimum score of all the indicators.

$$
\mathrm{M}=\operatorname{Min} M_{i} \quad \text { (2) }
$$

2) If the status of each indicator belongs to normal or attention, the final score of the device is the weighted sum of all the indicators.

$$
\mathrm{M}=\sum_{i=1}^{n} M_{i} \times C_{i}
$$

And finally determine the equipment status according to the scoring interval of the scoring interval, and predict the equipment failure. If the score is low, the maintenance can be arranged preferentially to detect the problem early and keep the equipment safe and stable.

\subsubsection{Entropy method calculation process}

Taking the line as an example, the weight value of the consideration dimension is calculated by the entropy method. First of all, get all kinds of raw data at the time of failure, then according to the index calculation formula for comprehensive score calculation, the following results are obtained: 
Table 1 Indicator score results

\begin{tabular}{|l|l|l|l|l|l|}
\hline $\begin{array}{l}\text { Serial } \\
\text { number }\end{array}$ & Occur time & Line load rate & $\begin{array}{l}\text { Terminal } \\
\text { on-line rate }\end{array}$ & $\begin{array}{l}\text { The correct rate } \\
\text { of remote action }\end{array}$ & $\begin{array}{l}\text { Line } \\
\text { connection rate }\end{array}$ \\
\hline 1 & $2016 / 05 / 0312: 13: 16$ & 0.9594 & 1 & 1 & 0.7272 \\
\hline 2 & $2016 / 05 / 1313: 13: 11$ & 0.7967 & 0.9 & 1 & 0.9090 \\
\hline 3 & $2016 / 05 / 2212: 15: 16$ & 0.8725 & 1 & 0.821 & 0.4545 \\
\hline 4 & $2016 / 06 / 1212: 34: 45$ & 0.9907 & 1 & 1 & 0.4533 \\
\hline 5 & $2016 / 06 / 1913: 13: 16$ & 0.9146 & 1 & 0.9205 & 1 \\
\hline 6 & $2016 / 07 / 0315: 13: 16$ & 0.9586 & 1 & 0.6367 & 0.8183 \\
\hline 7 & $2016 / 07 / 1216: 13: 38$ & 0.9531 & 1 & 0.5468 & 0.6325 \\
\hline 8 & $2016 / 07 / 2314: 23: 16$ & 0.8236 & 1 & 1 & 1 \\
\hline 9 & $2016 / 08 / 0311: 13: 16$ & 0.9191 & 1 & 0.9070 & 0.9090 \\
\hline 10 & $2016 / 08 / 0909: 13: 16$ & 0.9097 & 1 & 0.923 & 0.5426 \\
\hline 11 & $2016 / 09 / 0305: 43: 23$ & 0.6718 & 1 & 0.8185 & 1 \\
\hline
\end{tabular}

Standardize the results of the indicators, the use of the formula is as follows:

$$
\mathrm{x}_{i j}^{\prime}=\left(\frac{x_{i j}-\min \left(x_{1 j}, x_{2 j}, \ldots, x_{n j}\right)}{\max \left(x_{1 j}, x_{2 j}, \ldots, x_{n j}\right)-\min \left(x_{1 j}, x_{2 j}, \ldots, x_{n j}\right)}\right) \times 100
$$

$\mathrm{x}_{i j}^{\prime}$ is the normalized result of the value of the $\mathrm{j}$-th indicator in the $\mathrm{i}$-th event.

Calculate the index entropy:

$$
\begin{gathered}
p_{i j}=\frac{\mathrm{x}_{i j}^{\prime}}{\sum_{i=1}^{n} \mathrm{x}_{i j}^{\prime}}(\mathrm{i}=1,2, \ldots, \mathrm{n}, \mathrm{j}=1,2, \ldots, \mathrm{m}) \\
e_{j}=-\ln (n)^{-1} \sum_{i=1}^{n} p_{i j} \ln \left(p_{i j}\right)
\end{gathered}
$$

The results of the entropy are as follows:

Table 2 Index entropy results

\begin{tabular}{|l|l|l|l|l|}
\hline $\begin{array}{l}\text { Indicator } \\
\text { item }\end{array}$ & Line load rate & $\begin{array}{l}\text { Terminal on-line } \\
\text { rate }\end{array}$ & $\begin{array}{l}\text { The correct rate of } \\
\text { remote action }\end{array}$ & $\begin{array}{l}\text { Line connection } \\
\text { rate }\end{array}$ \\
\hline Entropy & 3.34 & 1.98 & 2.13 & 3.67 \\
\hline
\end{tabular}

Calculate information utility value:

$$
g_{j}=\frac{1-e_{j}}{m-E_{e}} \quad\left(E_{e}=\sum_{j=1}^{m} e_{j} \quad 0 \leq g_{j} \leq 1 \quad \sum_{j=1}^{m} g_{j}=1\right)
$$

Calculate the weight of the indicator:

$$
w_{j}=\frac{g_{j}}{\sum_{j=1}^{m} g_{j}}(1 \leq \mathrm{j} \leq \mathrm{m})
$$

The final weight value is as follows:

Table 3 Indicator Weights Results

\begin{tabular}{|l|l|l|l|l|}
\hline Indicator item & Line load rate & $\begin{array}{l}\text { Terminal on-line } \\
\text { rate }\end{array}$ & $\begin{array}{l}\text { The correct rate of } \\
\text { remote action }\end{array}$ & $\begin{array}{l}\text { Line connection } \\
\text { rate }\end{array}$ \\
\hline Weight value & 0.216 & 0.337 & 0.298 & 0.149 \\
\hline
\end{tabular}

\subsubsection{Model example}

Take the line as an example, obtain the complete historical data from the realtime database, combine the state index of the line at that time, and then establish the following line scoring model according to the importance of the various types of state indicators. 
Table 4 Index scoring model

\begin{tabular}{|l|l|l|l|l|l|}
\hline \multicolumn{2}{|c|}{ Fault level } & \multicolumn{3}{l|}{ Decentralized weight } \\
\cline { 4 - 7 } Status indicators and weights & & normal & attention & abnormal & serious \\
\hline Line load rate & 0.216 & 1 & 0.75 & 0.5 & 0.25 \\
\hline Terminal on-line rate & 0.337 & 1 & 0.75 & 0.5 & 0.25 \\
\hline Correct rate of remote action & 0.298 & 1 & 0.75 & 0.5 & 0.25 \\
\hline Line connection rate & 0.149 & 1 & 0.75 & 0.5 & 0.25 \\
\hline
\end{tabular}

If the current line state load rate is in the state of attention, the correct rate of remote action is attention, the other is normal, then the final score of the line:

$\mathrm{M}=0.216 * 100 * 0.75+0.337 * 100 * 1+0.298 * 100 * 0.75+0.149 * 100 * 1=87.15$

It belongs to the normal state, but need to strengthen the attention, so as to avoid problems.

If the current line state load rate is abnormal state, the other is normal, then the final score of the line:

$\mathrm{M}=100 * 0.5=50$

It belongs to the abnormal state, it may fail at any time, there need to arrange maintenance in time to ensure the safe and stable operation of the line.

\section{Summary}

This paper studies the effective integration of realtime database and D5200 system, realizes the function of changing and recording, improves the accuracy of distribution data, and can better server all kinds of upper application. This paper also studies the health state detection and diagnosis technology of distribution network equipment based on realtime database, which can find the weak links of the distribution network equipment, locate the fault quickly and give the processing opinions, and can predict the occurrence of possible failures. It can prevent the occurrence of failure and improve the efficiency of troubleshooting and ensure the safety of power supply.

\section{Reference}

[1] Feifei Wang. Research on Optimization of Distribution Network Maintenance Scheduling[D]. North China Electric Power University.2013.

[2] Jungao Huang, Shouding Wang, Qiang Ling, et al. Application of Time Sequenced Database In Regional Grid Dispatch and Control Integration System[J]. Automation of Electric Power Systems. 2011,24(23):107-111.

[3] Qilin Li. Research on Enterprise Application Integration Technology for Intelligent Power Grid[J]. Compute Sciense. 2011, 38(7):83-84

[4] Mingze Guo, Jianmin Zhang, Wendao Fang. Application of Heterogeneous System Data Integration Technology in DMS[J]. Information Technology. 2011, (8):173-176

[5] Junjie Hu. Researches and Applications of Smart Distribution Grid Information System Integration[D]. Zhejiang University.2011

[6] Minglei Huo. Research on Condition Maintenance and Decision Optimization of Power Distribution Equipment[D]. North China Electric Power University. 2015

[7] Yan Tang. Research on Condition Maintenance Decision of Distribution Network[D]. North China Electric Power University. 2014.

[8] Long Xu. Condition Assessment on Distribution Network Equipment[D]. North China Electric Power University. 2013. 
[9] Aiwu Zhou, Yafei Yu. Research on K-Means Clustering Algorithm[J]. Computer Technology and Development. 2011(2):62-65.

[10]Seng Ouyang, Yili Shi. Improved Entropy Method and Its Application in Power Quality Assessment[J]. Automation of Electric Power Systems. 2013(21): 156-159,164 